\title{
Upgrading of Resolution Elastic Neutron Scattering (RENS)
}

\author{
S. Magazù, F. Migliardo, and M. T. Caccamo \\ Department of Physics and Earth Sciences, University of Messina, Viale F. S. D’Alcontres 31, 98166 Messina, Italy \\ Correspondence should be addressed to S. Magazù; smagazu@unime.it
}

Received 31 May 2013; Accepted 18 July 2013

Academic Editor: Debashis Mukherji

Copyright (C) 2013 S. Magazù et al. This is an open access article distributed under the Creative Commons Attribution License, which permits unrestricted use, distribution, and reproduction in any medium, provided the original work is properly cited.

\begin{abstract}
An update of the Resolution Elastic Neutron Scattering (RENS) approach consisting in measuring the elastically scattered neutron intensity versus the instrumental energy resolution is presented. In particular it is shown that the measured elastic scattering law as a function of the logarithm of the instrumental energy of resolution gives rise to an increasing sigmoid trend whose inflection point can be connected with the system relaxation time. The validity of the RENS approach is supported by a numerical simulation, taking into account a Gaussian resolution function and a Lorentzian scattering law, and experimentally by integrated EINS and QENS measurements performed as a function of temperature on three homologous disaccharide/water mixtures showing different relaxation times. Furthermore, the most important advantages of the RENS approach are discussed; in particular, in comparison with QENS, the RENS approach requires a smaller amount of sample, which is an important point in dealing with biological and exotic systems, is not affected by the use of model functions for fitting spectra, and furnishes a direct access to the system relaxation time.
\end{abstract}

\section{Introduction}

The characterization of the molecular processes involved in condensed matter systems can be performed by Elastic Incoherent Neutron Scattering (EINS) by means of the so-called "fixed-windows" method [1], where the scattered intensity is collected at $\omega=0$ with a fixed "energy windows" corresponding to the instrumental energy resolution. In the case of purely elastic scattering, all the scattered neutrons fall within this energy window and the measured intensity remains constant at all temperatures, a part from the small decrease, due to the Debye-Waller factor, while changes in the elastically scattered intensity are registered when the system relaxation time becomes shorter than the instrumental resolution time. This technique takes advantage from the fact that, besides a relatively low number of fitting parameters, with respect to the quasielastic contribution at low energy transfer the elastic one is often a factor 100-1000 higher, and then for obtaining good quality data in reasonable times, due to the usually limited neutron fluxes, one can cope with a relatively small amount samples, with small sized samples, or with strongly absorbing samples. In this framework, the analysis of the EINS intensity, collected by varying some internal or external parameters, can provide useful information. For example, a wide wave-vector range (e.g., such as that of the back-scattering spectrometer IN13 at the ILL in Grenoble, with a $Q$ range extending up to $4.28 \AA^{-1}$ ) can allow achieving an assignment based on spatial characteristics; in other words, the elastic intensity versus momentum exchange can allow us to characterize molecular motion according to their spatial extent and amplitude. Furthermore, a varying spectral resolution and energy window increase the capability of deciphering the nature of the recorded relaxations on the basis of characteristic time. Moreover, the use of hydrogendeuterium labeling, highlighting contributions from specific scatterer groups or, separately, from protein and solvent, adds a dimension that can be crucial for spectral separation. Finally the temperature dependence of the measured elastic intensity can provide important clues on the activation energies and, thus, on the local potentials; in fact, for example, the guiding picture for a water soluble protein is that increasing temperature from the lowest values where only vibrations mainly contribute, one reveals some more or less marked variations in intensity or in some intensity derived physical quantities, such as the Mean Square Displacement (MSD), that may correspond to the "activation" of some internal motions (e.g., methyl groups rotations), to motions induced 
by solvent fluctuations, to the internal protein $\mathrm{H}$-bonds, to the kinetic melting of solvent, to the melting of bulk solvent, and so forth [2-6].

It is well known that the time dependent pair correlation function $G(r, t)$ represents the probability to find a particle at distance $r$ after a time $t$ when a particle was at $t=0$ in $r=0$. The scattering law $S(Q, \omega)$ and the intermediate scattering function $I(Q, t)$ are directly connected, in Planck's units, through time and space Fourier transforms. As a matter of fact, the previously introduced functions make reference to the system properties and not to measured quantities; in fact, it should be taken into account that, for example, when the experimental technique gives access to the scattering law one should take into account the less straightforward connection with the measured scattering function, which is the convolution of the scattering law with the instrumental resolution function and that can be expressed as the time Fourier transform of the product between the Intermediate Scattering Function and the Resolution function in the time domain.

The aim of this work is to present an updated approach, that is, RENS, which is based on the acquisition of the measured elastic scattering law as a function of the instrumental energy resolution. In particular, the effect of the coupling between the system and the employed measuring instrument is considered and it is shown that the measured elastic scattering law as a function of the logarithm of the instrumental energy resolution behaves like an increasing sigmoid curve whose inflection point occurs when this instrumental resolution time matches the system relaxation time.

In order to test the validity of the RENS approach we shall consider three systems constituted by water mixtures of three homologous disaccharides, that is, trehalose, maltose, and sucrose; these systems are shown, by QENS measurements, to exhibit different dynamics as a function of temperature; as a consequence when tested by EINS measurements at a given instrumental energy resolution as a function of temperatures, these systems are supposed to show, following the RENS approach, different inflection point temperatures. The collection of the measured elastic scattering intensity versus temperature, showing different inflection points for the three investigated systems, confirms the validity of the approach.

\section{Materials and Methods}

Ultrapure powdered trehalose, maltose and sucrose, $\mathrm{D}_{2} \mathrm{O}$ and $\mathrm{H}_{2} \mathrm{O}$, purchased by Aldrich-Chemie, were used for the experiment.

EINS data were collected in the 20-310 K temperature range on the three homologous disaccharides trehalose, maltose, and sucrose in $\mathrm{H}_{2} \mathrm{O}$ at weight fraction values corresponding to 19 water molecules for each disaccharide molecule by the IN13 spectrometer at ILL (Grenoble, France). The used IN13 configuration is the following; incident wavelength: $2.23 \AA$, Q-range: $0.28-4.27 \AA^{-1}$. Raw data were corrected for cell scattering and detector response and normalized to unity at $Q=0.00 \AA^{-1}$.
Quasielastic neutron scattering (QENS) measurements were performed in a temperature range of $283-320 \mathrm{~K}$ on hydrogenated trehalose, maltose, and sucrose $\left(\mathrm{C}_{12} \mathrm{H}_{22} \mathrm{O}_{11}\right)$ in $\mathrm{H}_{2} \mathrm{O}$ and on partially deuterated trehalose, maltose, and sucrose $\left(\mathrm{C}_{12} \mathrm{H}_{14} \mathrm{D}_{8} \mathrm{O}_{11}\right)$ in $\mathrm{D}_{2} \mathrm{O}$ at weight fraction values corresponding to 19 water $\left(\mathrm{H}_{2} \mathrm{O}\right.$ and $\left.\mathrm{D}_{2} \mathrm{O}\right)$ molecules for each disaccharide molecule. In order to obtain partially deuterated samples, the disaccharides were first dissolved in pure $\mathrm{D}_{2} \mathrm{O}$ at a concentration of $\sim 40 \%$ by weight, to exchange the eight labile hydrogen atoms of the disaccharides and the solutions were, subsequently, lyophilized. The procedure was repeated in order to allow the exchange of all the exchangeable hydrogens. The solution samples were obtained by dissolution of the $\mathrm{D}_{2} \mathrm{O}$-exchanged lyophilized disaccharides in pure $\mathrm{D}_{2} \mathrm{O}$. The QENS experiment was carried out by the IRIS at ISIS (RAL, UK). We used the high resolution configuration of IRIS (graphite 002 and mica 006 analyser reflections) to measure sets of QENS spectra covering a $Q, \omega$-domain extending from $\hbar \omega=-0.3$ to $0.6 \mathrm{meV}$ (energy transfer) and $Q=0.3$ to $1.8 \AA^{-1}$ (momentum transfer). The detectors used give a mean energy resolution of $\Gamma=8 \mu \mathrm{eV}$ of Half Width at Half Maximum (HWHM) as determined by reference to a standard vanadium plate.

\section{Results and Discussion}

It is well known that the scattering law $S(Q, \omega)$ and the intermediate scattering function $I(Q, t)$ are connected by a time Fourier transform [7-12]:

$$
\begin{aligned}
& S(Q, \omega)=\frac{1}{2 \pi} \int_{-\infty}^{+\infty} I(Q, t) e^{-i \omega t} d t \\
& I(Q, t)=\frac{1}{2 \pi} \int_{-\infty}^{+\infty} S(Q, \omega) e^{i \omega t} d \omega .
\end{aligned}
$$

The frequency instrumental resolution function $R(Q, \omega)$ in the $\omega$ space is connected to the corresponding time instrumental resolution function by the following Fourier transform:

$$
R(Q, t)=\frac{1}{2 \pi} \int_{-\infty}^{+\infty} R(Q, \omega) e^{i \omega t} d \omega
$$

The resolution scattering law corresponds to the convolution of the scattering law:

$$
S_{R}(Q, \omega)=S(Q, \omega) \otimes R(Q, \omega)
$$

Therefore, from a formal point of view, the convolution of the two functions $S(Q, \omega)$ and $R(Q, \omega)$ produces a third function $S_{R}(Q, \omega)$ which can be viewed as a modified version of the original function $S(Q, \omega)$, given by the overlap area between the two functions, that is, the $S(Q, \omega)$ and the $\omega$ inverted $R(Q, \omega)$ functions when this latter is translated. As a consequence for instrumental resolution functions $R(Q, \omega)$ symmetric in $\omega$, the convolution coincides with the crosscorrelation between $S(Q, \omega)$ and $R(Q, \omega)$. 
By the convolution theorem (3) can be written as

$$
\begin{aligned}
S_{R}(Q, \omega) & =S(Q, \omega) \otimes R(Q, \omega) \\
& =\frac{1}{\sqrt{2 \pi}} \int_{-\infty}^{+\infty} I(Q, t) \cdot R(Q, t) e^{-i \omega t} d t .
\end{aligned}
$$

In the ideal case of purely elastic scattering in which the resolution function is a delta in the $\omega$-space and, hence, a constant in the $t$-space, we obtain that having:

$$
S_{R}(Q, \omega=0)=\frac{1}{\sqrt{2 \pi}} \int_{-\infty}^{+\infty} I(Q, t) \cdot R(Q, t) d t
$$

the measured scattering law $S_{R}(Q, \omega)$ reduces to a time average of the intermediate scattering function $I(Q, t)$.

A part from this ideal case, in the more general condition in which the resolution function in the $\omega$-space has a nonnegligible width, the experimentally measured elastic scattering law, due to the finite energy instrumental resolution $\Delta \omega$, $S_{R}^{M}(Q)$ is

$$
S_{R}^{M}(Q)=\int_{-\Delta \omega / 2}^{+\Delta \omega / 2} S_{R}(Q, \omega) d \omega,
$$

and hence,

$$
\begin{aligned}
S_{R}^{M}(Q) & =\int_{-\Delta \omega / 2}^{+\Delta \omega / 2} S(Q, \omega) \otimes R(Q, \omega) d \omega \\
& =\int_{-\Delta \omega / 2}^{+\Delta \omega / 2}\left[\frac{1}{\sqrt{2 \pi}} \int_{-\infty}^{+\infty}(I(Q, t) \cdot R(Q, t)) e^{-i \omega t} d t\right] d \omega .
\end{aligned}
$$

In order to show the operation approach of RENS as well as the expected experimental output we numerically test the behaviour of the measured elastic scattering function versus the instrumental energy resolution. On this purpose we operate in the frequency space and evaluate $S_{R}^{M}(Q)$ as a function of the energy instrumental resolution $\Delta \omega$, in the case in which the scattering law $S(Q, \omega)$, is a Lorentzian curve with a fixed linewidth (as, for example, it occurs for a simple Brownian translational diffusion process) and the resolution function $R(Q, \omega)$ is a Gaussian curve. In particular, in order to numerically get the RENS output we consider an increasing instrumental resolution function linewidth $\Delta \omega$ and apply (7) for determining the value of the definite integral as a function of $\Delta \omega$. Figure 1 shows the result of such a calculation, that is, $S_{R}^{M}$ as a function of the logarithm of the instrumental resolution. As it can be seen it shows an increasing sigmoid trend whose inflection point occurs when the linewidth of the resolution function approaches the linewidth of the system scattering law; in other words, in such a semilogarithmic plot the inflection point occurs when the instrumental resolution time crosses the system relaxation time. Such a result shows the operating way of the RENS approach: from the inflection point of EINS profiles versus the logarithm of the energy resolution one can extract the system relaxation time. It should be noticed that, in a complementary way, for a given fixed instrumental energy resolution function from EINS

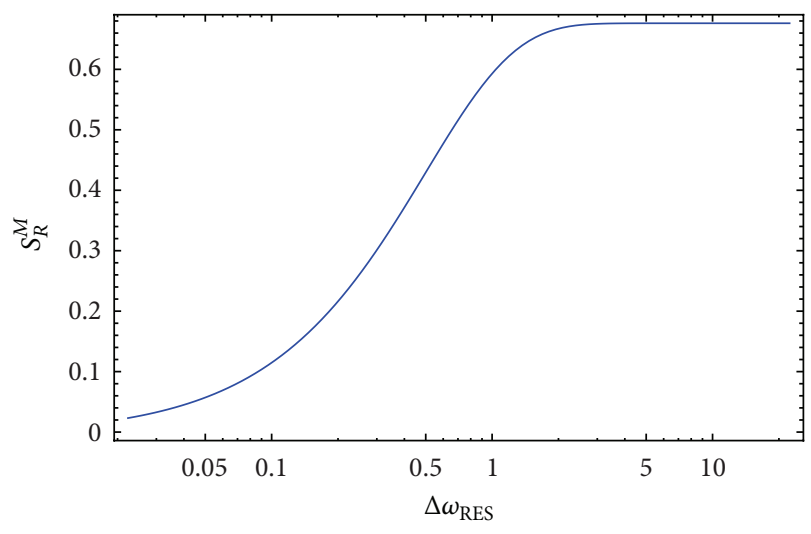

FIGURE 1: $S_{R}^{M}$ as a function of the logarithm of the instrumental resolution; the inflection point of the increasing sigmoid trend can be connected with the system relaxation time.

profiles versus temperature one is able to obtain from the inflection point the temperature value for which the system relaxation time equals the resolution time. In the following we will apply such a procedure to demonstrate the validity of the RENS approach.

On the other hand, as far as the system cross-section is concerned, it is well known that the neutron scattering double differential cross-section can be written as

$$
\begin{aligned}
& \frac{d^{2} \sigma}{d \Omega d \omega}=\frac{k_{1}}{k_{0}} \frac{1}{2 \pi} \int_{-\infty}^{+\infty} d t e^{-i \omega t} \\
& \times \frac{1}{N} \sum_{\alpha=1}^{N} \sum_{\beta=1}^{N}\left\langle b_{\alpha}^{*} b_{\beta} e^{-i \mathbf{Q} \cdot \mathbf{R}_{\alpha}(0)} e^{-i \mathbf{Q} \cdot \mathbf{R}_{\beta}(t)}\right\rangle \\
& =\frac{k_{1}}{k_{0}} \frac{1}{2 \pi} \int_{-\infty}^{+\infty} d t e^{-i \omega t} \frac{1}{N} \\
& \times\left[|\bar{b}|^{2} \sum_{\alpha} \sum_{\alpha \neq \beta}\left\langle e^{-i \mathbf{Q} \cdot \mathbf{R}_{\alpha}(0)} e^{-i \mathbf{Q} \cdot \mathbf{R}_{\beta}(t)}\right\rangle\right. \\
& \left.+\overline{|b|^{2}} \sum_{\alpha}\left\langle e^{-i \mathbf{Q} \cdot \mathbf{R}_{\alpha}(0)} e^{-i \mathbf{Q} \cdot \mathbf{R}_{\alpha}(t)}\right\rangle\right] \\
& =\frac{k_{1}}{k_{0}}|\bar{b}|^{2} S_{\text {coh }}(Q, \omega)+\frac{k_{1}}{k_{0}} \overline{|b|^{2}} S_{\text {inc }}(Q, \omega) \\
& +\frac{k_{1}}{k_{0}}\left(|\bar{b}|^{2}-\overline{|b|^{2}}\right) S_{\text {inc }}(Q, \omega) \\
& =\frac{k_{1}}{k_{0}}|\bar{b}|^{2} S(Q, \omega)+\frac{k_{1}}{k_{0}}\left(|\bar{b}|^{2}-\overline{|b|^{2}}\right) S_{\text {inc }}(Q, \omega) \\
& =\frac{k_{1}}{k_{0}} \frac{\sigma_{\mathrm{coh}}}{4 \pi} S(Q, \omega)+\frac{k_{1}}{k_{2}} \frac{\sigma_{\text {inc }}}{4 \pi} S_{\text {inc }}(Q, \omega) \\
& =\frac{k_{1}}{k_{0}}\left[\frac{\sigma_{\mathrm{coh}}}{4 \pi} S(Q, \omega)+\frac{\sigma_{\mathrm{inc}}}{4 \pi} S_{\mathrm{inc}}(\mathrm{Q}, \omega)\right] \text {, }
\end{aligned}
$$




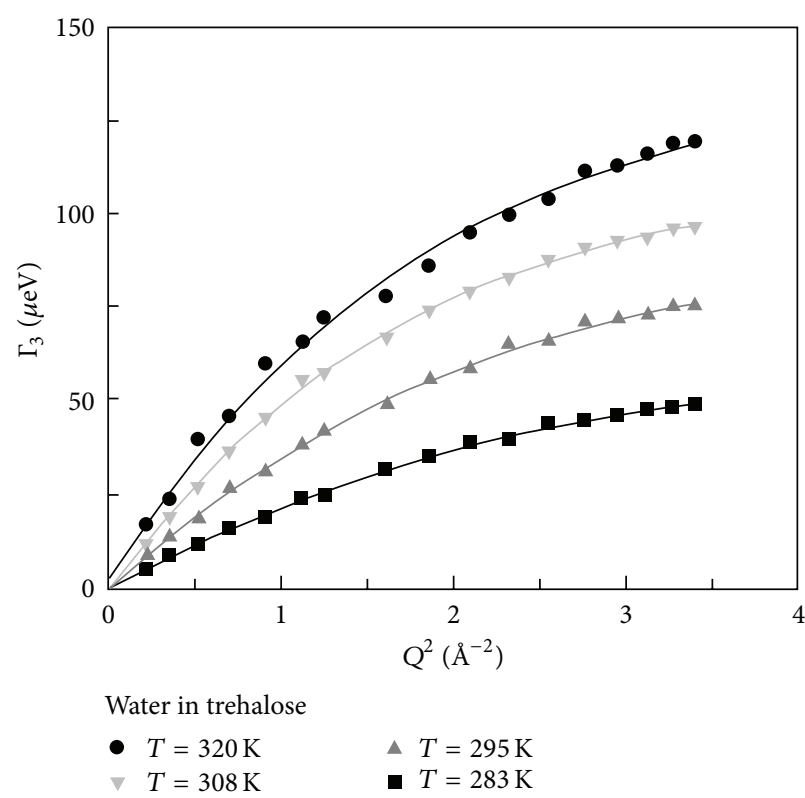

(a)

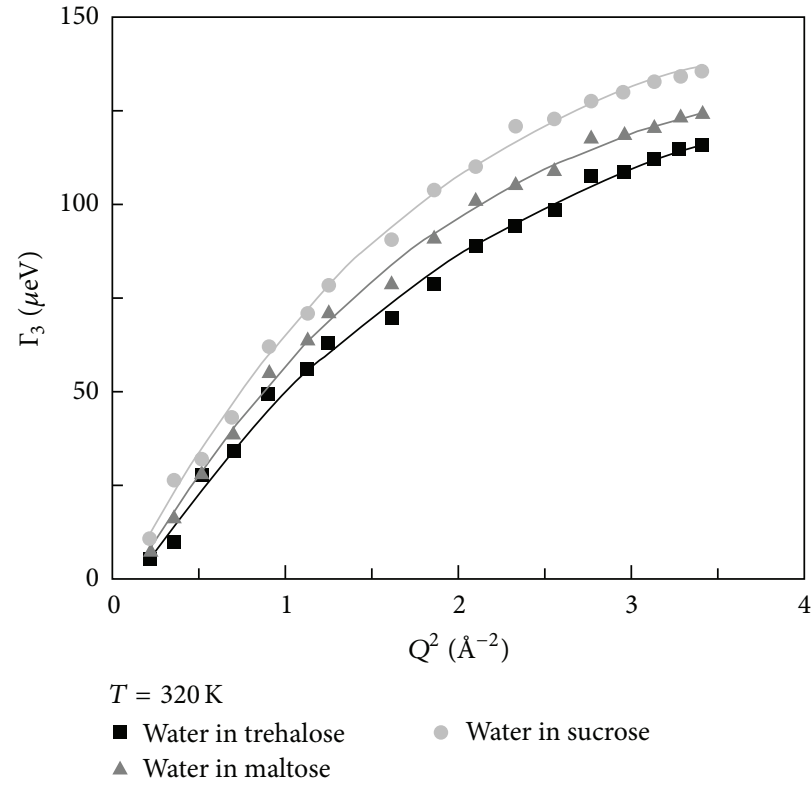

(b)

Figure 2: Translational linewidth $\Gamma_{3}$ of (a) water in trehalose solutions for different temperature values and of (b) water in trehalose, maltose, and sucrose aqueous solutions at $T=320 \mathrm{~K}$ as a function of $Q^{2}$.

where $k_{0}$ and $k_{1}$ are the incoming and outcoming neutron wavevectors, $b_{\alpha}$ and $b_{\beta}$ are the scattering lengths of atom $\alpha$ and $\beta$, respectively, and it is $|\bar{b}|^{2}=\left\langle b_{\alpha}^{*} b_{\beta}\right\rangle, \overline{|b|^{2}}=\left\langle b_{\alpha}^{*} b_{\alpha}\right\rangle, \sigma_{\text {coh }}=$ $4 \pi|\bar{b}|^{2}$, and $\sigma_{\text {inc }}=4 \pi\left(|\bar{b}|^{2}-|\bar{b}|^{2}\right) . \sigma_{\text {coh }}$ takes into account interference effects among waves produced by the scattering of a single neutron from all the nuclei, whereas $\sigma_{\text {inc }}$ refers to single particle properties.

In our case, due to the high percentage of hydrogen atoms in the investigated systems $\left(\mathrm{H}: \sigma_{\text {coh }}=1,76 ; \sigma_{\text {inc }}=80,26\right)$ only this latter contribution is relevant:

$$
\frac{d^{2} \sigma}{d \Omega d \omega} \cong \frac{k_{1}}{k_{0}}\left[\frac{\sigma_{\mathrm{inc}}}{4 \pi} S_{\mathrm{inc}}(Q, \omega)\right] .
$$

In the following, the QENS and EINS data on disaccharides water mixtures are presented and discussed in the framework of the above-described RENS approach.

The analysis performed on the QENS data of disaccharide $/ \mathrm{D}_{2} \mathrm{O}$ and disaccharide $/ \mathrm{H}_{2} \mathrm{O}$ mixtures [13-16] allows us to characterise the diffusive dynamics of both solute and solvent. By determining the translational contribution, its linewidth $\Gamma$ for disaccharides and water as a function of $Q^{2}$ follows a typical Random Jump Diffusion (RJD) model [9]:

$$
\Gamma_{1}(Q)=\frac{D_{s} Q^{2}}{1+D_{s} Q^{2} \tau},
$$

where $D_{s}$ is the self-diffusion coefficient of the molecule and $\tau$ is the residence time. The RJD model furnishes the diffusion coefficient $D_{s}$ value from the extrapolation to $Q \rightarrow 0$ and the residence time $\tau$ from the inverse of the asymptotic value at $Q \rightarrow \infty$.
Such a model furnishes for trehalose the diffusion coefficient $D_{s}$ and the residence time $\tau$ of $D_{s}=2.83 \times 10^{-7} \mathrm{~cm}^{2} / \mathrm{s}$ and $\tau=24.7 \mathrm{ps}, D_{s}=3.82 \times 10^{-7} \mathrm{~cm}^{2} / \mathrm{s}$ and $\tau=20.6 \mathrm{ps}, D_{\mathrm{s}}=$ $5.35 \times 10^{-7} \mathrm{~cm}^{2} / \mathrm{s}$ and $\tau=19.1 \mathrm{ps}$, and $D_{s}=8.50 \times 10^{-7} \mathrm{~cm}^{2} / \mathrm{s}$ and $\tau=18.3$ ps for $T=283,295,308$, and $320 \mathrm{~K}$, respectively.

The RJD model furnishes for the diffusion coefficient $D_{s}$ and the residence time $\tau$ the values of $D_{s}=8.50 \times 10^{-7} \mathrm{~cm}^{2} / \mathrm{s}$ and $\tau=18.3 \mathrm{ps}$ for trehalose, $D_{s}=1.00 \times 10^{-6} \mathrm{~cm}^{2} / \mathrm{s}$ and $\tau=14.3 \mathrm{ps}$ for maltose, and $D_{s}=1.23 \times 10^{-6} \mathrm{~cm}^{2} / \mathrm{s}$ and $\tau=$ $13.8 \mathrm{ps}$ for sucrose at $T=320 \mathrm{~K}$.

The best fit according to the RJD model for the translational linewidth $\Gamma_{3}$ of water in trehalose solutions at different temperature values and of water in trehalose, maltose, and sucrose aqueous solutions at $T=320 \mathrm{~K}$ as a function of $Q^{2}$ is reported in Figure 2. The diffusion coefficient of water in the three disaccharide aqueous solutions at $T=320 \mathrm{~K}$ are: $D_{w}=8.31 \times 10^{-6} \mathrm{~cm}^{2} / \mathrm{s}$ for trehalose solution, $D_{w}=$ $8.46 \times 10^{-6} \mathrm{~cm}^{2} / \mathrm{s}$ for maltose solution, and $D_{w}=8.60 \times$ $10^{-6} \mathrm{~cm}^{2} / \mathrm{s}$ for sucrose solution, with the values of residence times of $\tau=3.7 \mathrm{ps}, 3.4 \mathrm{ps}$, and $3.0 \mathrm{ps}$ for trehalose, maltose and sucrose solutions, respectively. It should be noticed that the diffusion coefficient of pure water at the same temperature is $D_{w}=3.94 \times 10^{-5} \mathrm{~cm}^{2} / \mathrm{s}$.

For the investigated systems the water dynamics resembles that of water at $\sim 268 \mathrm{~K}$ in the case of trehalose solution, at $\sim 271 \mathrm{~K}$ in the case of maltose solution, and at $\sim 277 \mathrm{~K}$ in the case of sucrose solution, while the whole water dynamics in trehalose solutions for $T=283,295,308$, and $320 \mathrm{~K}$ resembles that of water at $\sim 256 \mathrm{~K}, \sim 261 \mathrm{~K}, \sim 263 \mathrm{~K}$, and $\sim$ $268 \mathrm{~K}$, indicating that the water has a diffusive behaviour strongly triggered by the trehalose molecules and suffers from a noticeable frozen effect. Analogously to the trehalose aqueous solutions, all the disaccharides show a slowing down 


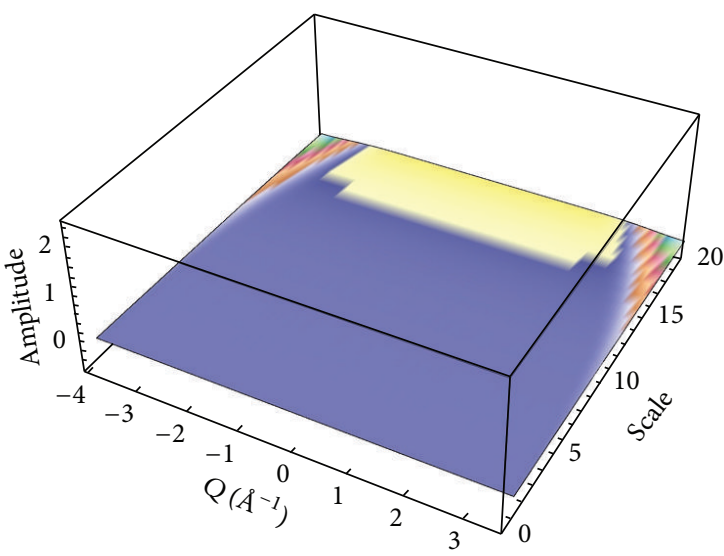

(a)

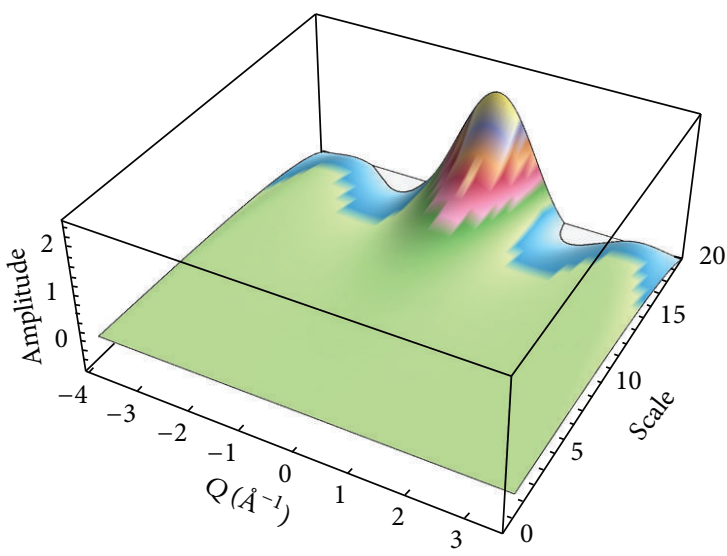

(c)

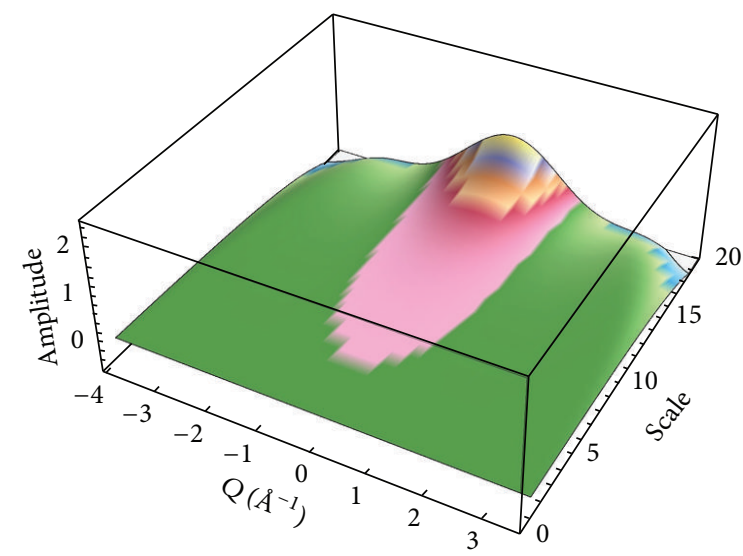

(b)

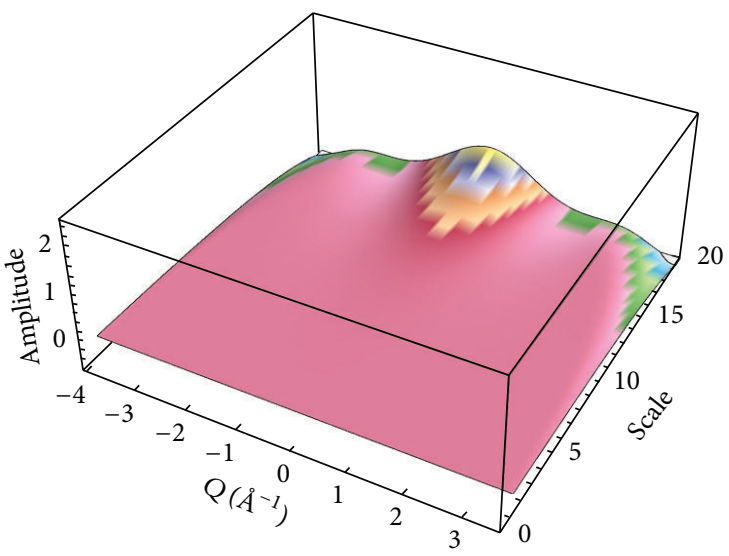

(d)

FIGURE 3: 3D scalograms of a wavelet analysis for sucrose/water mixtures, at three different temperatures, (a) $T=19 \mathrm{~K}$, (b) $T=264 \mathrm{~K}$, and (c) $T=284 \mathrm{~K}$, and (d) $3 \mathrm{D}$ scalogram of a wavelet analysis for trehalose/water mixtures at $T=264$.

effect of the water dynamics, which is stronger for trehalose than the other disaccharides.

The higher slowing down effect of the diffusive dynamics observed for trehalose is evidently linked to its extraordinary capability to "switch off" the metabolic functions.

The EINS data on the same systems, that is, disaccharide $/ \mathrm{H}_{2} \mathrm{O}$ mixtures [17-21], give an experimental confirmation of the RENS approach. In particular, the experimental findings have been analyzed by means of a wavelet analysis that allows us to characterize the scattered elastic intensity behavior on different scales highlighting the correlation between the signal and the set of the scaled and translated mother functions [22]. From a formal point of view, a continuous wavelet transform can be written as

$$
W_{I}(\tau, s)=\int_{-\infty}^{\infty} I(t) \psi_{\tau, s}^{*}(t) d t,
$$

where $\tau \in \mathbb{R}$ is the translation parameter, whereas $s \in$ $\mathbb{R}^{+}(s \neq 0)$ is the scale parameter, $I(t)$ is the function to be analyzed, the asterisks denote the complex conjugate, and $\psi_{\tau, s}$ is the mother wavelet:

$$
\psi_{\tau, s}=\frac{1}{\sqrt{s}} \psi\left(\frac{t-\tau}{s}\right)
$$

the factor $s^{-1 / 2}$ is an adimensional normalization constant.
More precisely, the translated-version allow the wavelets to be localized, whereas the scaled-version wavelets allows us to analyze the signal in different scales through the so-called scalogram, that is, the square of the modulus of the wavelet transform:

$$
P_{W_{I}}(Q, s)=\left|W_{I}(Q, s)\right|^{2} .
$$

More in details, the result of a wavelet analysis of the EINS data is shown in Figure 3, which reports the 3D scalograms at $T=264 \mathrm{~K}$. Such an analysis allows us to compare both the spatial properties of the three systems in the wavevector range of $Q=0.27 \AA^{-1} \div 4.27 \AA^{-1}$.

More precisely, starting from the lowest investigated temperature, $19 \mathrm{~K}$, the presence of only one spectral contribution extending in the whole investigated wavevector range is revealed. Such a wide and flat contribution spans the whole wavevector range and can be attributed to the vibrational motions of the scatterer particles, that is, the system's protons. As it can be seen in Figure 3, by increasing temperature a different contribution, at low wavevector values, clearly emerges. As pointed out by the adopted chromatic scales for the wavelet coefficients, already at this temperature value such a contribution is much less marked in the case of 
TABLE 1: Parameters of the fitting procedure according to (14) of the EINS intensity as a function of temperature for the three disaccharides.

\begin{tabular}{|c|c|c|c|c|c|}
\hline Disaccharide & Amplitude & Steepness & Intercept & Slope & Temperature \\
\hline Trehalose & 0,00391 & 0,14678 & 0,01669 & $1,47 E-05$ & 264 \\
\hline Maltose & 0,00832 & 0,16482 & 0,01628 & $2,70 E-05$ & 259 \\
\hline Sucrose & 0,00963 & 0,18184 & 0,01618 & $3,42 E-05$ & 257 \\
\hline
\end{tabular}

trehalose. Finally the 3D scalograms of sucrose, maltose, and trehalose water mixtures at $T=284 \mathrm{~K}$ reveal that the weight of this latter contribution tends to increase with temperature showing a different increasing rate for the three investigated systems. Therefore, the EINS results confirm that the dynamics of the trehalose/water system is slower with respect to that of the maltose/water and of the sucrose/water systems.

In order to test the validity of the RENS approach one should take into account that, since the three systems exhibit different relaxation times at all the investigated temperatures, when tested by EINS at a given instrumental energy resolution as a function of temperatures, they should show different inflection point temperatures.

In order to extract quantitative information from the $I(T)$ behaviors we performed a fit by using the following expression:

$$
I(T)=A\left(1-\frac{1}{1+e^{-B\left(T-T_{0}\right)}}\right)+(C-D T) .
$$

Here $A$ represents the amplitude factor connected with the global thermal intensity behavior, whose inverse we call "thermal restrain"; it should be noticed that this quantity is not connected with a local behavior but represents a global property of the system. $B$ is connected with the transition steepness, passing from smaller to higher values when the transition becomes more and more abrupt. As it can be inferred by following the RENS approach, $T_{0}$ is the inflection temperature, corresponding to the temperature value for which the system relaxation time equals the instrumental resolution time [4]. On this concern such a temperature value should occur at different values for the different disaccharides. Finally, $C-D T$ represents the low temperature vibrational contribution.

In Figure 4 the case of maltose intensity versus temperature is shown as an example while in the insert the behavior for the three disaccharides is reported. The results of the fitting procedure of EINS intensity as a function of temperatures for the three disaccharides performed by applying (14) are reported in Table 1. As it can be seen, the analysis indicates a higher thermal restrain together with a higher transition temperature for trehalose with respect to the other two homologous disaccharides. Such a result confirms the validity of the RENS approach. In fact, taking into account the meaning of the transition temperature, the disaccharideswater relaxation times cross the resolution time at different temperature values, that is, $264 \mathrm{~K}$ for trehalose, $259 \mathrm{~K}$ for maltose, and $257 \mathrm{~K}$ for sucrose.

In conclusion, this contribution shows the operating approach of RENS from an analysis performed on the frequency space: from the inflection point of EINS profiles

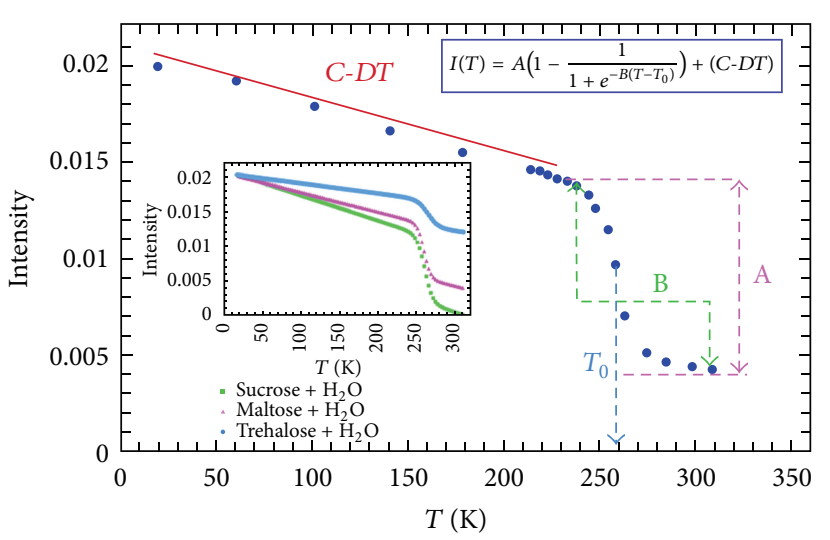

FIgURE 4: Intensity profile, obtained as sum for all the wavevector values, for maltose $/ \mathrm{H}_{2} \mathrm{O}$ versus temperature with the fitting result according to (14).

versus the logarithm of the energy resolution one can extract the system relaxation time. In a complementary way, for a given fixed instrumental energy resolution function from EINS profiles versus temperature one is able to obtain from the inflection point the temperature value for which the system relaxation time equals the resolution time.

\section{References}

[1] M. Prager, W. Press, B. Alefed, and A. Huller, "Rotational states of the $\mathrm{NH}_{4}^{+}$ion in $\left(\mathrm{NH}_{4}\right)_{2} \mathrm{SnCl}_{6}$ by inelastic neutron scattering," Journal of Chemical Physics, vol. 67, no. 11, p. 5126, 1977.

[2] A. Lerbret, G. Lelong, P. E. Mason, M.-L. Saboungi, and J. W. Brady, "Molecular dynamics and neutron scattering study of glucose solutions confined in MCM-41," Journal of Physical Chemistry B, vol. 115, no. 5, pp. 910-918, 2011.

[3] G. Lelong, R. Heyd, G. Charalambopoulou et al., "Role of glucose in enhancing stability of aqueous silica gels against dehydration," Journal of Physical Chemistry C, vol. 116, no. 17, pp. 9481-9486, 2012.

[4] A. Lerbret, P. E. Mason, R. M. Venable et al., "Molecular dynamics studies of the conformation of sorbitol," Carbohydrate Research, vol. 344, no. 16, pp. 2229-2235, 2009.

[5] G. Lelong, W. S. Howells, J. W. Brady, C. Talón, D. L. Price, and M.-L. Saboungi, "Translational and rotational dynamics of monosaccharide solutions," Journal of Physical Chemistry B, vol. 113, no. 39, pp. 13079-13085, 2009.

[6] L. Lupi, L. Comez, M. Paolantoni, D. Fioretto, and B. M. Ladanyi, "Dynamics of biological water: insights from molecular modeling of light scattering in aqueous trehalose solutions," The Journal of Physical Chemistry B, vol. 116, pp. 7499-7508, 2012. 
[7] L. van Hove, "Correlations in space and time and Born approximation scattering in systems of interacting particles," Physical Review, vol. 95, no. 1, pp. 249-262, 1954.

[8] F. Volino, Spectroscopic Methods for the Study of Local Dynamics in Polyatomic Fluids, Plenum, New York, NY, USA, 1978.

[9] M. Bee, Quasielastic Neutron Scattering, Adam Hilger, Bristol, UK, 1988.

[10] S. Magazù, "NMR, static and dynamic light and neutron scattering investigations on polymeric aqueous solutions," Journal of Molecular Structure, vol. 523, pp. 47-59, 2000.

[11] A. Rahman, K. S. Singwi, and A. Sjölander, "Theory of slow neutron scattering by liquids. I," Physical Review, vol. 126, no. 3, pp. 986-996, 1962.

[12] S. Magazù, G. Maisano, F. Migliardo et al., "Characterization of molecular motions in biomolecular systems by elastic incoherent neutron scattering," Journal of Chemical Physics, vol. 129, no. 15, Article ID 155103, 2008.

[13] S. Magazù, F. Migliardo, and M. T. F. Telling, "Study of the dynamical properties of water in disaccharide solutions," European Biophysics Journal, vol. 36, no. 2, pp. 163-171, 2007.

[14] S. Magazù, F. Migliardo, and M. T. F. Telling, " $\alpha, \alpha$-trehalosewater solutions. VIII. Study of the diffusive dynamics of water by high-resolution quasi elastic neutron scattering," The Journal of Physical Chemistry B, vol. 110, no. 2, pp. 1020-1025, 2006.

[15] S. Magazù, F. Migliardo, and M. T. F. Telling, "Structural and dynamical properties of water in sugar mixtures," Food Chemistry, vol. 106, no. 4, pp. 1460-1466, 2008.

[16] F. Affouard, P. Bordat, M. Descamps et al., "A combined neutron scattering and simulation study on bioprotectant systems," Chemical Physics, vol. 317, no. 2-3, pp. 258-266, 2005.

[17] I. V. Blazhnov, S. Magazù, G. Maisano, N. P. Malomuzh, and F. Migliardo, "Macro- and microdefinitions of fragility of hydrogen-bonded glass-forming liquids," Physical Review E, vol. 73, no. 3, Article ID 031201, 7 pages, 2006.

[18] S. Magazù, F. Migliardo, C. Mondelli, and M. Vadalà, "Correlation between bioprotective effectiveness and dynamic properties of trehalose-water, maltose-water and sucrose-water mixtures," Carbohydrate Research, vol. 340, no. 18, pp. 2796-2801, 2005.

[19] S. Magazù, F. Migliardo, C. Mondelli, and G. Romeo, “Temperature dependence of mean square displacement by IN13: a comparison between trehalose and sucrose water mixtures," Chemical Physics, vol. 292, pp. 247-251, 2003.

[20] S. Magazù, G. Maisano, and F. Migliardo, "Fragility by elastic incoherent neutron scattering," Journal of Chemical Physics, vol. 121, no. 18, pp. 8911-8915, 2004.

[21] S. Magazù, G. Maisano, F. Migliardo, and C. Mondelli, “ $\alpha, \alpha-$ trehalose/water solutions. VII: an elastic incoherent neutron scattering study on fragility," The Journal of Physical Chemistry $B$, vol. 108, no. 36, pp. 13580-13585, 2004.

[22] S. Magazù, F. Migliardo, and M. T. Caccamo, "Innovative wavelet protocols in analyzing elastic incoherent neutron scattering," The Journal of Physical Chemistry B, vol. 116, no. 31, pp. 94179423, 2012. 

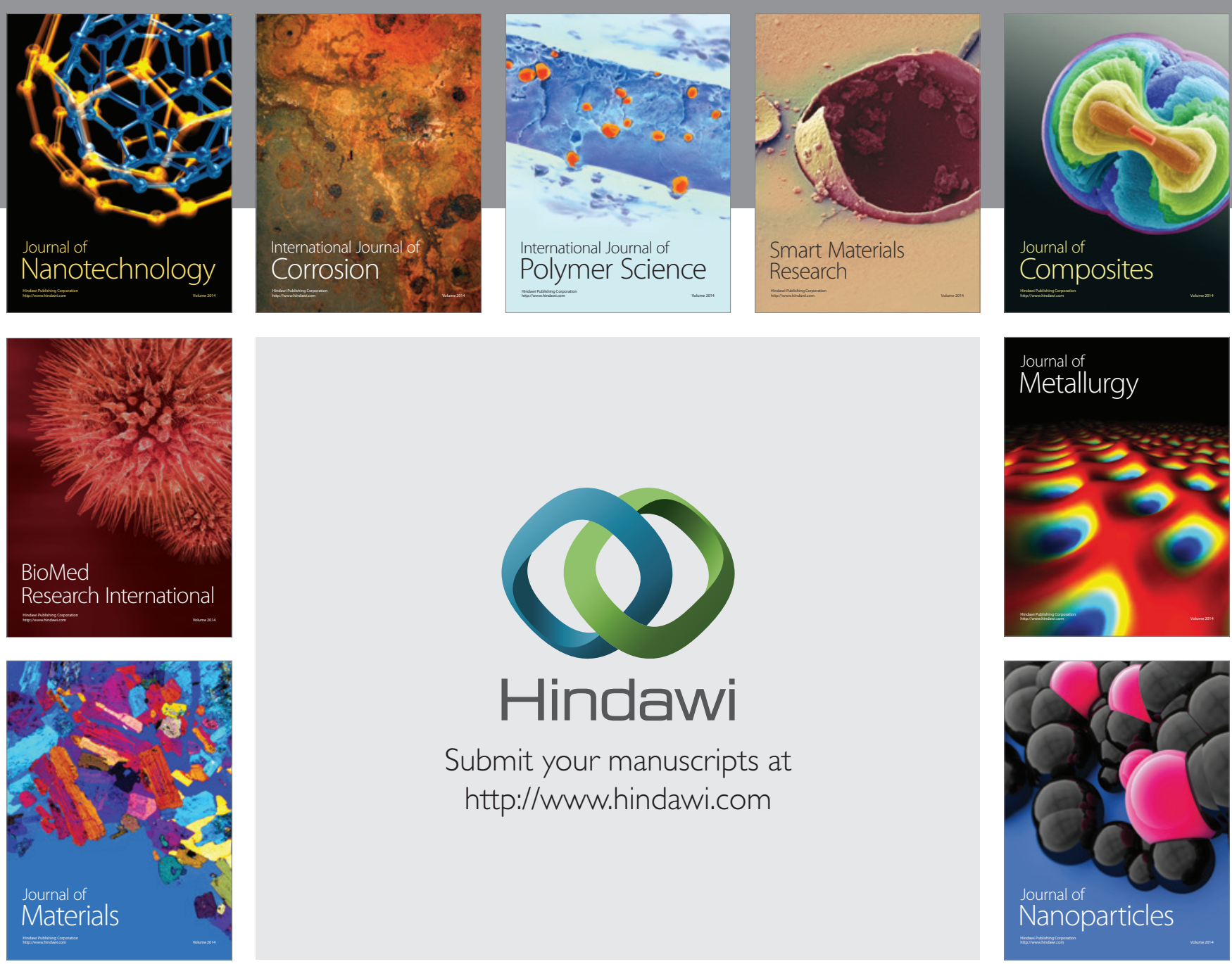

Submit your manuscripts at http://www.hindawi.com
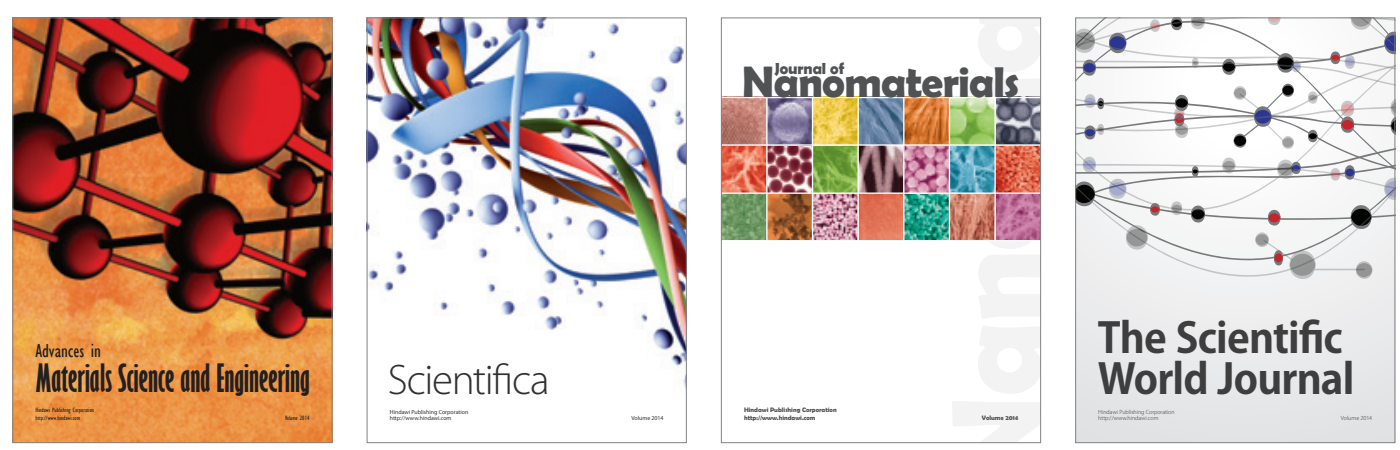

\section{The Scientific World Journal}
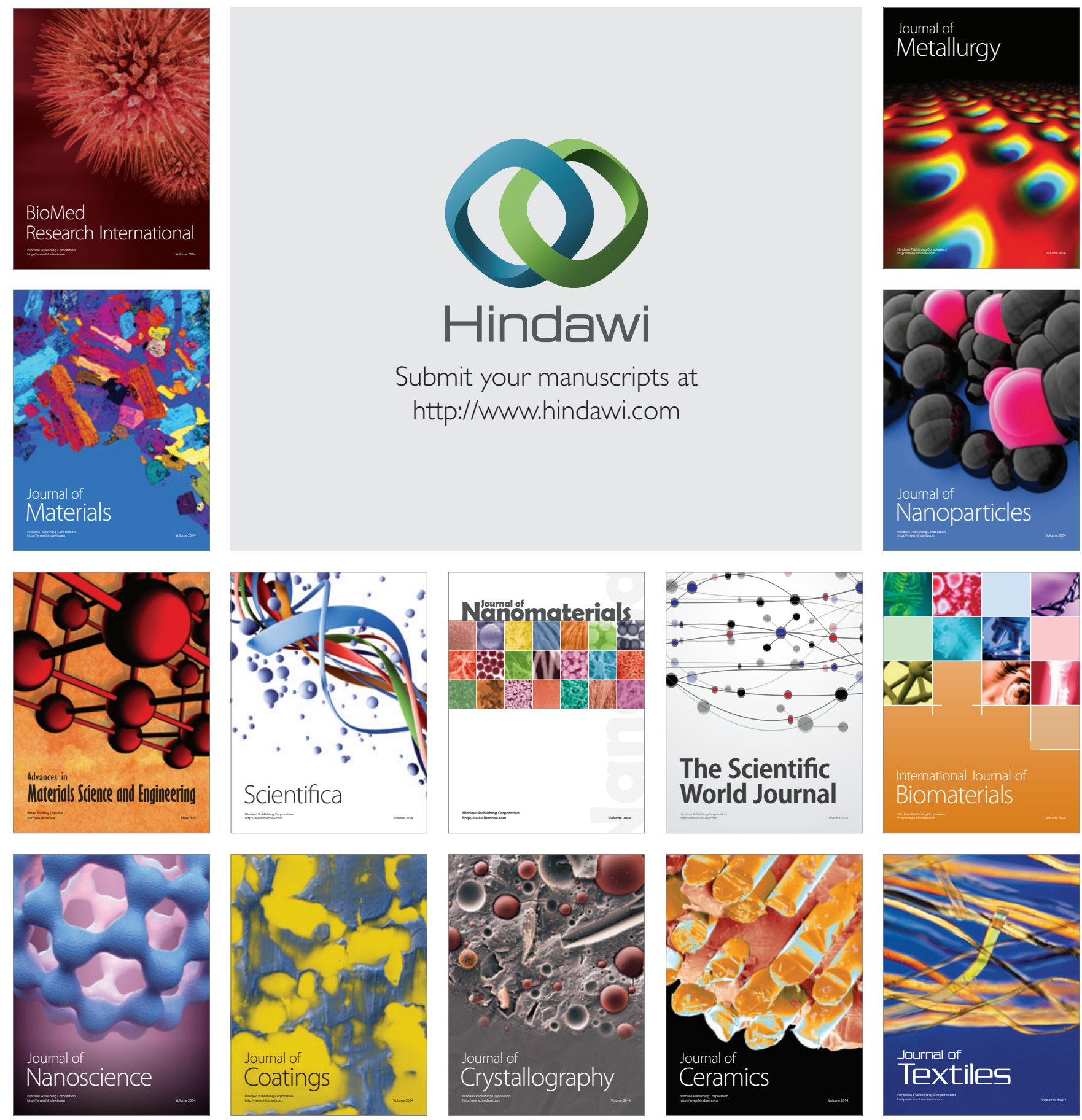